\title{
From the Topical Issue Editor
}

Received: 13 June 2012 / Accepted: 16 June 2013

(C) 3D Research Center, Kwangwoon University and Springer 2013

$\mathrm{W}$ elcome to the topical issue of 3D RESEARCH on "Multi-view Videoplus-Depth Coding."

As the technologies of digital image processing advance, the demands for interactive contents are dramatically increasing. We can reproduce natural and realistic $3 \mathrm{D}$ scenes by combining the

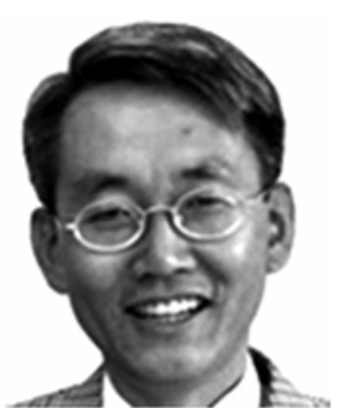
depth data with the conventional 2D scene for threedimensional television (3DTV). In 3DTV, we can generate virtual views that provide us with unrestricted spatiotemporal navigation by employing multi-view videoplus-depth (MVD) data. Since the MVD data require a huge amount of data to be stored or transmitted to the user in proportional to the number of cameras, we need to develop efficient coding methods for such applications.

Multi-view video coding (MVC) has been studied earlier than multi-view video-plus-depth coding (MVD). In October 2004, it was reported that multi-view video coding algorithms gave much better results than simulcast coding with H.264/AVC. As a result, the joint multi-view video model based on the joint scalable video model was proposed and reference software for multi-view video coding was released in October 2006. In April 2007, the MVD format was presented for advanced future video systems, such as 3DTV and FTV. After finalizing the standardization on MVC in 2008, experts raised a request of a new coding standard that can provide free-viewpoint video generation at the decoder with low complexity. As a result, the MVD data format was employed as a new 3D video format. Until now, multi-view video-plus-depth coding technologies have been under investigation by the joint collaborative team on 3D video coding (JCT-3V).
Yo-Sung Ho ( $\bowtie)$

School of Information and Communication, Gwangju Institute of Science and Technology, Gwangju, South Korea

TEL: +82- 62-970-2211

Web site: https://infcom1.gist.ac.kr/eng/persons/view/professor/29 E-mail: hoyo@gist.ac.kr
The contributions of this issue cover various aspects of 3DTV, especially focusing on multi-view video-plus-depth coding. Takanori Senoh et al. presented a multi-view image coding algorithm which was composed of a global depth generator, residual view generator, core codec of MPEG HEVC, and a view synthesizer. Cheon Lee et al. proposed an efficient depth map coding method using a residual segmentation that divided residual data into two regions with a segment map and two mean values, instead of using transformation. Dmytro Rusanovskyy et al. described a novel approach of using depth information for advanced coding of associated video data in 3D video systems.

Other aspects of 3DTV are also covered. Lingfeng Xu et al. proposed a ray-space interpolation algorithm to generate virtual viewpoint images in the epipolar plane images based on Radon transform. Shuqiang Yang et al. presented a novel and stable human detection and behavior recognition method based on an obliquely mounted depth camera for intelligent surveillance systems, consisting of four parts: depth camera calibration, digital elevation map generation, human detection and tracking, and human behavior recognition. Mohd Shafry Mohd Rahim et al. performed evaluation of an adaptive subdivision method as a benchmark for further research on increasing capabilities of subdivision, especially on mobile devices of very limited resources in term of memory and processing.

We hope that you enjoy this collection of papers describing recent progress on MVD coding and other aspects of 3DTV. We would like to thank all authors, reviewers, and staffs in Springer for supporting this topical issue.

\section{Yo-Sung Ho}

Topical Issue Editor 